\title{
Polyols and glucose particulate species as tracers of primary biogenic organic aerosols at 28 french sites
}

Abdoulaye Samake ${ }^{1}$, Jean-Luc Jaffrezo ${ }^{1}$, Olivier Favez ${ }^{2}$, Samuël Weber ${ }^{1}$, Véronique Jacob ${ }^{1}$, Alexandre Albinet ${ }^{2}$, Véronique Riffault ${ }^{3}$, Esperanza Perdrix ${ }^{3}$, Antoine Waked ${ }^{1 *}$, Benjamin Golly ${ }^{1}$, Dalia Salameh ${ }^{1+}$, Florie Chevrier ${ }^{1}$, Diogo Miguel Oliveira ${ }^{2,3}$, Jean-Luc Besombes ${ }^{4}$, Jean M.F Martins ${ }^{1}$, Sébastien Conil ${ }^{5}$, Géraldine Guillaud ${ }^{6}$, Boualem Meshba ${ }^{7}$, Benoit Rocq ${ }^{8}$, Pierre-Yves Robic ${ }^{9}$, Agnès Hulin ${ }^{10}$, Sébastien Le Meur $^{11}$, Maxence Descheemaecker ${ }^{12}$, Eve Chretien ${ }^{13}$, and Gaëlle Uzu ${ }^{1}$.

${ }^{1}$ Univ. Grenoble Alpes, CNRS, IRD, INP-G, IGE (UMR 5001), F-38000 Grenoble, France.

${ }^{2}$ INERIS, Parc Technologique Alata, BP 2, 60550 Verneuil-en-Halatte, France

${ }^{3}$ IMT Lille Douai, Univ. Lille, SAGE - Département Sciences de l'Atmosphère et Génie de l’Environnement, F-59000 Lille, France

${ }^{4}$ Univ. Savoie Mont-Blanc, LCME, F-73000 Chambéry, France

${ }^{5}$ ANDRA DRD/GES Observatoire Pérenne de l'Environnement, F-55290 Bure, France

${ }^{6}$ Atmo AuRA, F-38400 Grenoble, France

${ }^{7}$ Air PACA, F-03040, France

${ }^{8}$ Atmo Hauts de France, F-59000, France

${ }^{9}$ Atmo Occitanie, F-31330 Toulouse, France

${ }^{10}$ Atmo Nouvelle Aquitaine, F-33000, France

${ }^{11}$ Atmo Normandie, F-76000, France

${ }^{12}$ Lig'Air, F-45590 Saint-Cyr-en-Val, France

${ }^{13}$ Atmo Grand Est, F-16034 Strasbourg, France

*Now at IMT Lille Douai, Univ. Lille, SAGE - Département Sciences de l'Atmosphère et Génie de I'Environnement, F-59000 Lille, France

${ }^{\dagger}$ Now at Airport pollution control authority (ACNUSA), 75007 Paris, France

Corresponding author(s): A Samaké (abdoulaye.samake2@univ-grenoble-alpes.fr) and JL Jaffrezo (Jean-luc.Jaffrezo@univ-grenoble-alpes.fr) 
Table S1: Characteristics of sampling sites (including altitude, site typology) and number of analyzed samples

\begin{tabular}{|c|c|c|c|c|c|c|c|}
\hline Sampling site & $\begin{array}{l}\text { Altitude } \\
(\mathrm{m})\end{array}$ & $\begin{array}{c}\text { PM } \\
\text { fraction }\end{array}$ & $\begin{array}{l}\text { AASQA* in charge } \\
\text { of sampling sites }\end{array}$ & $\begin{array}{l}\text { Number of } \\
\text { Samples }\end{array}$ & $\begin{array}{c}\text { Sugar } \\
\text { compounds } \\
\text { analyzed by }\end{array}$ & $\begin{array}{c}\text { PMF } \\
\text { analysis }\end{array}$ & Typology \\
\hline OPE-ANDRA & 293 & $\mathrm{PM}_{10}$ & OPE-ANDRA & 266 & IGE & & Rural \\
\hline OPE-ANDRA & 293 & $\mathrm{PM}_{2.5}$ & OPE-ANDRA & 310 & IGE & & Rural \\
\hline Peyrusse Vieille & 175 & $\mathrm{PM}_{10}$ & ATMO Occitanie & 59 & IGE & & Rural \\
\hline Revin & 395 & $\mathrm{PM}_{10}$ & ATMO Grand-Est & 168 & LSCE & + & Rural \\
\hline Revin & 395 & $\mathrm{PM}_{2.5}$ & ATMO Grand-Est & 162 & IGE & & Rural \\
\hline Dieulefit & 550 & $\mathrm{PM}_{2.5}$ & ATMO AURA & 56 & IGE & & Rural \\
\hline Verneuil & 180 & $\mathrm{PM}_{2.5}$ & LIG'AIR & 60 & IGE & & Rural \\
\hline Chamonix & 1035 & $\mathrm{PM}_{10}$ & ATMO AuRA & 120 & IGE & + & Urban \\
\hline Marnaz & 504 & $\mathrm{PM}_{10}$ & ATMO AuRA & 203 & IGE & + & Urban \\
\hline Passy & 588 & $\mathrm{PM}_{10}$ & ATMO AuRA & 344 & IGE & + & Urban \\
\hline Lanslebourg & 1400 & $\mathrm{PM}_{10}$ & ATMO AuRA & 82 & IGE & & Urban \\
\hline Grenoble_LF & 214 & $\mathrm{PM}_{10}$ & ATMO AuRA & 714 & IGE & + & Urban \\
\hline Grenoble_CB & 212 & $\mathrm{PM}_{10}$ & ATMO AuRA & 72 & IGE & & Urban \\
\hline Grenoble_VIF & 310 & $\mathrm{PM}_{10}$ & ATMO AuRA & 72 & IGE & & Urban \\
\hline Gap & 743 & $\mathrm{PM}_{10}$ & AIR PACA & 125 & IGE & & Urban \\
\hline Lyon & 160 & $\mathrm{PM}_{10}$ & ATMO AuRA & 172 & IGE & + & Urban \\
\hline Marseille & 64 & $\mathrm{PM}_{10}$ & AIR PACA & 255 & IGE & + & Urban \\
\hline Gardanne & 212 & $\mathrm{PM}_{10}$ & AIR PACA & 88 & IGE & & Urban \\
\hline Meyreuil & 235 & $\mathrm{PM}_{10}$ & AIR PACA & 91 & IGE & & Urban \\
\hline Mallet & 200 & $\mathrm{PM}_{10}$ & AIR PACA & 96 & IGE & & Urban \\
\hline Port-de-Bouc & 1 & $\mathrm{PM}_{10}$ & AIR PACA & 242 & IGE & + & Urban \\
\hline Aix-en-Provence & 188 & $\mathrm{PM}_{10}$ & AIR PACA & 177 & IGE & + & Urban \\
\hline Nice & 9 & $\mathrm{PM}_{10}$ & AIR PACA & 228 & IGE & + & Urban \\
\hline Talence & 20 & $\mathrm{PM}_{10}$ & $\begin{array}{l}\text { ATMO Nouvelle- } \\
\text { Aquitaine }\end{array}$ & 159 & IGE & + & Urban \\
\hline Poitiers & 0 & $\mathrm{PM}_{10}$ & $\begin{array}{l}\text { ATMO Nouvelle- } \\
\text { Aquitaine }\end{array}$ & 134 & IGE & + & Urban \\
\hline Lens & 47 & $\mathrm{PM}_{10}$ & ATMO & 118 & IGE & + & Urban \\
\hline & & & Hauts-de-France & 169 & LSCE & + & Urban \\
\hline Nogent & 47 & $\mathrm{PM}_{10}$ & $\begin{array}{c}\text { ATMO } \\
\text { Hauts-de-France }\end{array}$ & 155 & LSCE & + & Urban \\
\hline Rouen & 6 & $\mathrm{PM}_{10}$ & ATMO Normandie & 168 & LSCE & + & Urban \\
\hline Roubaix & 10 & $\mathrm{PM}_{10}$ & $\begin{array}{c}\text { ATMO } \\
\text { Hauts-de-France }\end{array}$ & 159 & LSCE & + & Traffic \\
\hline Strasbourg & 139 & $\mathrm{PM}_{10}$ & $\begin{array}{c}\text { ATMO } \\
\text { Grand-Est }\end{array}$ & 120 & IGE & + & Traffic \\
\hline
\end{tabular}

*AASQA: Officially-approved French Air Quality Monitoring networks which instrument and maintain the sampling sites, and handle the filters. Symbol (+) indicates cases where PMF analysis was performed.

*Except PM 10 collected at OPE-ANDRA (sampled on weekly basis with low volume sampler operating at a flow rate of $\left.1 \mathrm{~m}^{3} . \mathrm{h}^{-1}\right)$, all others $P M$ samples were collected on daily basis, using high volume samplers operating at a flow rate of $30 \mathrm{~m}^{3} \cdot h^{-1}$. 


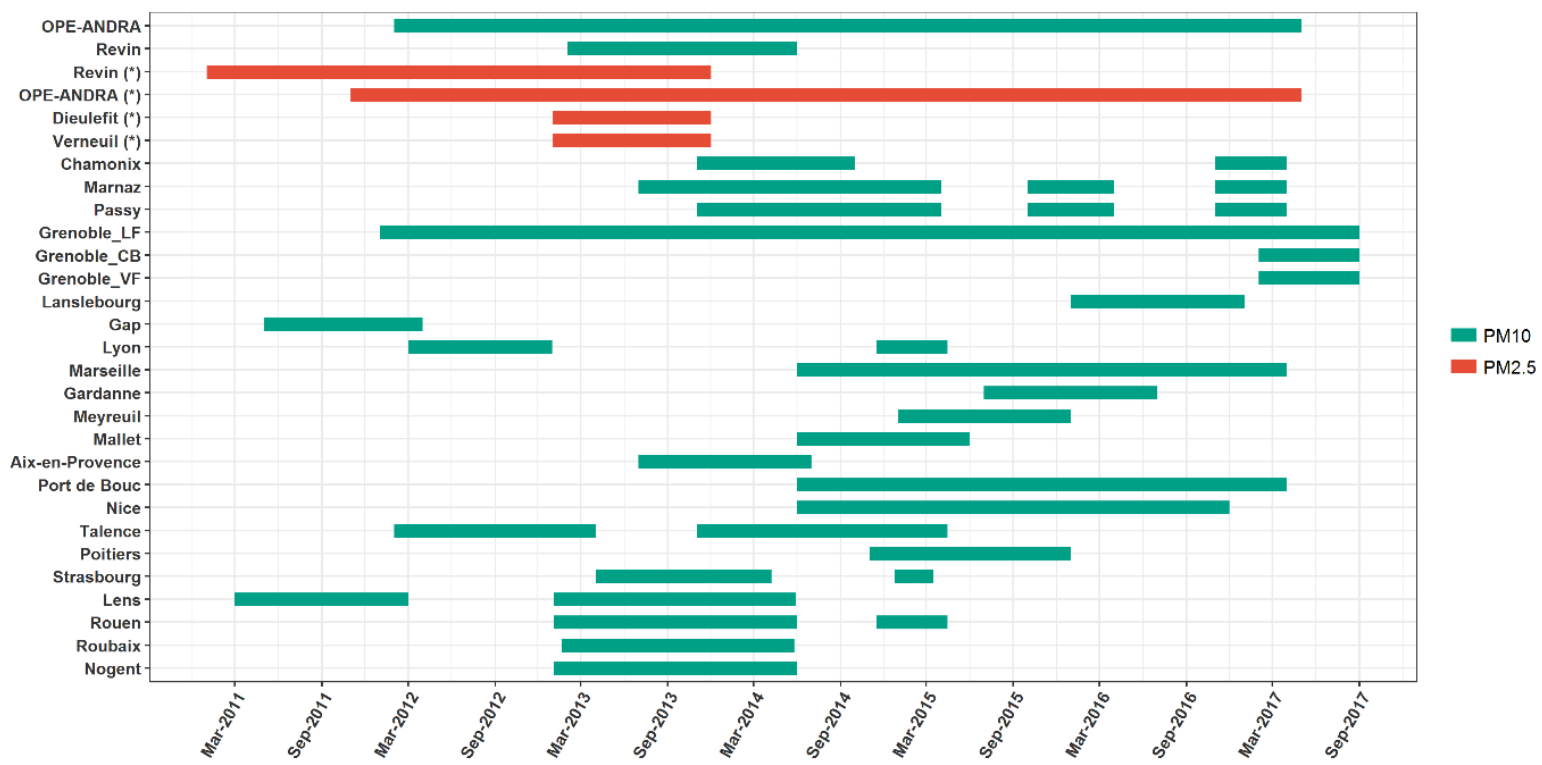

Figure S1: Timeline of Particulate matter (PM) sampling campaign periods for each studied site.

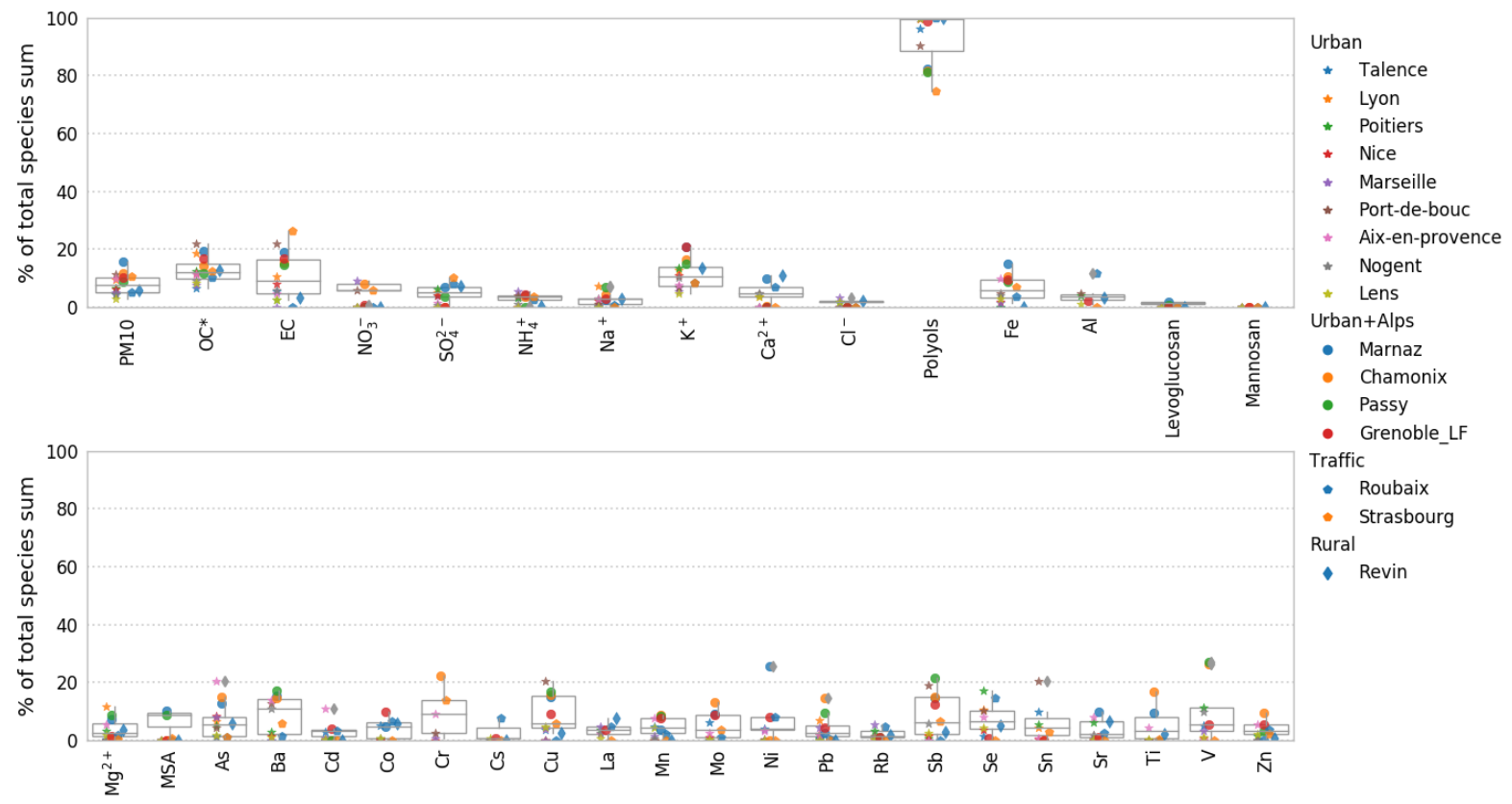

Figure S2: Percentage of each species apportioned by the PBOA profile from PMF studies. Values lower than a few pg $\mu g^{-1}$ are not displayed on purpose. For each boxplot, the top, middle and bottom lines of the box represent the $75^{\text {th }}$, median and $25^{\text {th }}$ percentile, respectively. The whiskers at the top and bottom of the box extend from the maximum to the minimum. 


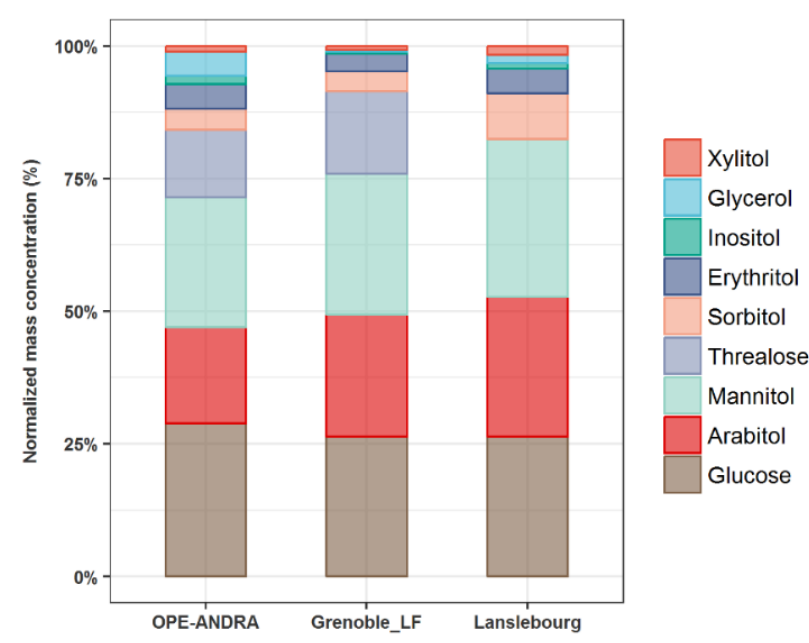

Figure S3: Overview of the average relative mass distributions of individual primary sugar alcohols and saccharide compounds quantified in some aerosol samples at three sites over summer and autumn periods (June to November) corresponding to maximal atmospheric concentrations of sugar alcohols/saccharide compounds. For Lanslebourg, threalose is not visible since its concentration was below the limit of quantification.

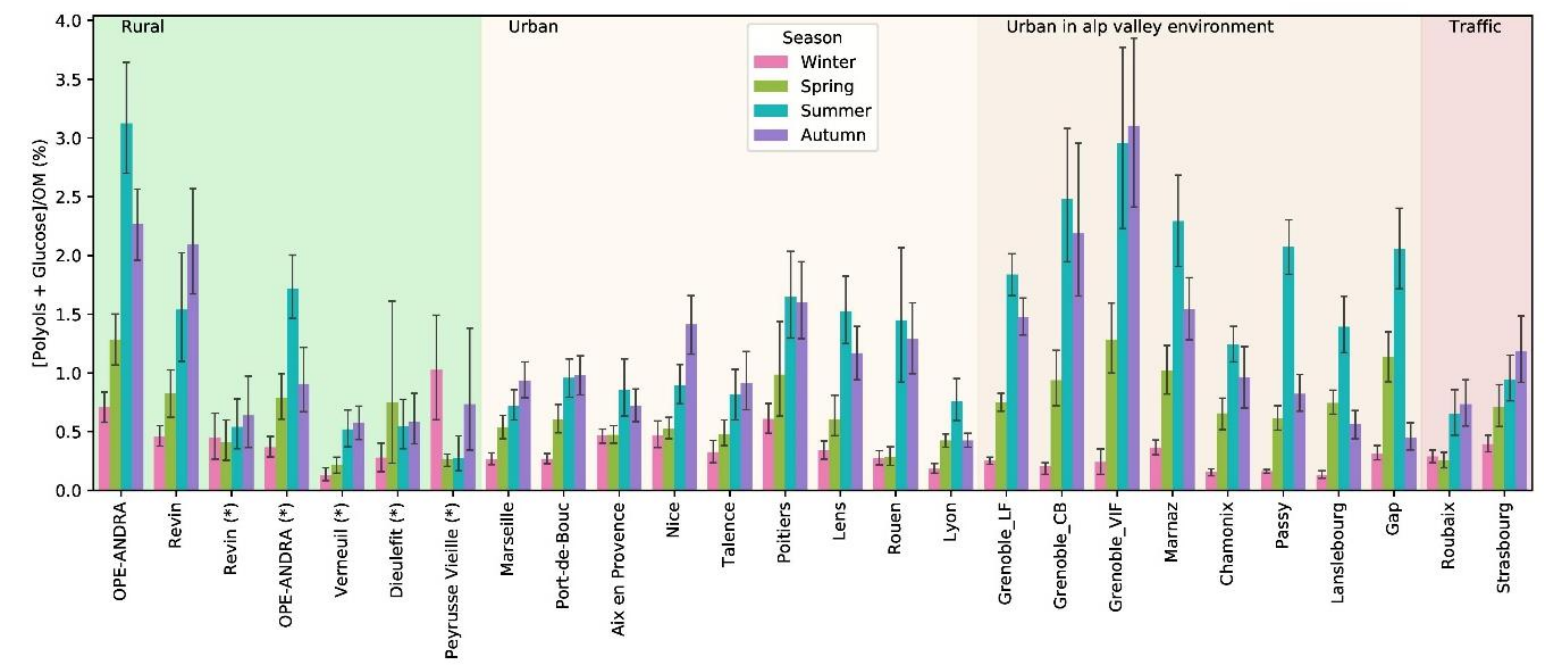

Figure S4: Spatial and seasonal average contributions of dominant polyols (arabitol + mannitol) and glucose to total organic (OM) aerosols at various sites in France. Symbol (*) indicates $P M_{2.5}$ aerosol samples 


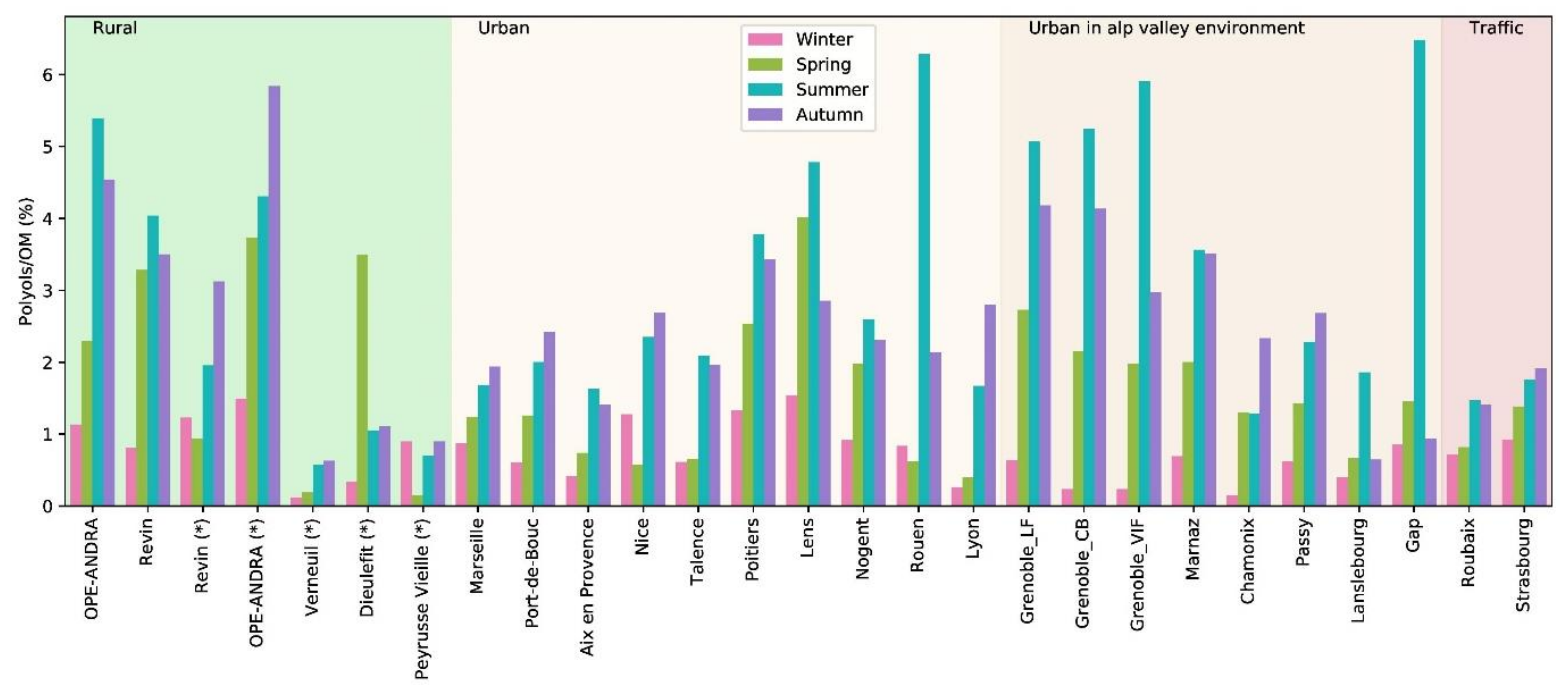

Figure S5 : Maximum seasonal contributions of dominant polyols to total organic aerosols (OM) at various sites in France. Symbol (*) indicates $P M_{2.5}$ aerosol samples. 
Table S2: PBOA average factor profile (in fraction of PM mass) identified in the DECOMBIO and SOURCES programs.

\begin{tabular}{|c|c|c|}
\hline Chemical species & Mean (ng $\mu g^{-1}$ ) & Standard deviation $\left(n g \mu^{-1}\right)$ \\
\hline OC* & 394 & 51 \\
\hline $\mathrm{EC}$ & 81 & 68 \\
\hline $\mathrm{Cl}^{-}$ & 3.9 & 6.8 \\
\hline $\mathrm{NO}_{3}^{-}$ & 22 & 49 \\
\hline $\mathrm{SO}_{4}{ }^{2-}$ & 55 & 61 \\
\hline $\mathrm{Na}^{+}$ & 4.6 & 5.2 \\
\hline $\mathrm{NH} 4^{+}$ & 13 & 14 \\
\hline $\mathrm{K}^{+}$ & 9.5 & 3.2 \\
\hline $\mathrm{Mg}^{2+}$ & 0.75 & 0.86 \\
\hline $\mathrm{Ca}^{2+}$ & 5.6 & 6.3 \\
\hline MSA & 0.12 & 0.32 \\
\hline Polyols & 25 & 10 \\
\hline Levoglucosan & 0.5 & 1.5 \\
\hline Mannosan & 0 & 0 \\
\hline $\mathrm{Fe}$ & 9.0 & 6.2 \\
\hline \multirow[t]{2}{*}{$\mathrm{Al}$} & 3.1 & 2.5 \\
\hline & $\left(p g \mu g^{-1}\right)$ & $\left(p g \mu g^{-1}\right)$ \\
\hline As & 21 & 19 \\
\hline $\mathrm{Ba}$ & 209 & 153 \\
\hline $\mathrm{Cd}$ & 1.8 & 3.2 \\
\hline Co & 3.2 & 4.7 \\
\hline $\mathrm{Cr}$ & 107 & 182 \\
\hline Cs & 0.6 & 1.5 \\
\hline $\mathrm{Cu}$ & 351 & 298 \\
\hline La & 5.4 & 4.8 \\
\hline $\mathrm{Mn}$ & 115 & 130 \\
\hline Mo & 11 & 11 \\
\hline $\mathrm{Ni}$ & 31 & 57 \\
\hline $\mathrm{Pb}$ & 75 & 80 \\
\hline $\mathrm{Rb}$ & 7 & 10 \\
\hline $\mathrm{Sb}$ & 29 & 26 \\
\hline Se & 45 & 54 \\
\hline Sn & 43 & 45 \\
\hline $\mathrm{Sr}$ & 31 & 36 \\
\hline $\mathrm{Ti}$ & 64 & 111 \\
\hline $\mathrm{V}$ & 32 & 33 \\
\hline $\mathrm{Zn}$ & 333 & 484 \\
\hline
\end{tabular}

OC* corresponds to the bulk organic carbon fraction minus individual molecular weight of characterized organic species. 